Acta Cryst. (1985). B41, 1-4

\title{
Checklist for Authors of Papers Submitted to Acta Crystallographica, Section B
}

Papers are considered for publication in Section B if they contribute to an advance in structural science. The following three criteria should be met:

1. The paper must contain a major structural element. This component may be an original determination of one or more structures (a single structure should generally have been studied under more than one condition of temperature or pressure), a theoretical structural investigation including new methodology, a study of structural relationships based on a search of the literature, or similar study.

2. The paper should also present an experimental and/or theoretical contribution to one of the natural sciences that is novel, original and of high quality.

3. The paper should combine these two types of contribution to provide new structural insight for that science or for crystallography.

In future, Lead Articles will be published on an occasional basis in Section B. Although Lead Articles may contain a review element, their primary emphasis will be with the direction in which an area of structural science is moving, indicating major problems, with suggestions for future development of the subject. Lead Articles will normally be invited but authors wishing to contribute such papers are asked to discuss their proposal with the Editor in advance. Such articles will be subject to normal review.

Attention is drawn to the Suggested guidelines for the publication of Rietveld analyses and pattern decomposition studies [J. Appl. Cryst. (1982). 15, 357359] and to Recommendations of the Ad-hoc Committee on Criteria for Publication of Charge Density Studies [J. Appl. Cryst. (1984). 17, 369]. Determinations based on powder data should be accompanied by copies of the data in the appropriate format \{see Notes for Authors [Acta Cryst. (1983). A39, 174-196], Appendix III\}; these will be deposited both with the JCPDS, Swarthmore, and at the Union's office in Chester. Papers on polytypes will be treated no differently from those on other materials and basic diffraction data relating to these should be made available either for publication or for deposition. See also Hamor, Steinfink \& Willis [Acta Cryst. (1985). C41, 301-303] for a summary of recommended techniques used in crystal structure determinations.

The checklist below is provided for the convenience of authors submitting papers for publication in Section B. Fuller information is given in Notes for Authors. Checklists for authors of papers submitted to Sections A and C of Acta Crystallographica and to Journal of Applied Crystallography are given in the first issue of those journals for 1985.

All papers submitted for publication in Acta Crystallographica, Section B, should be checked against the following list:

(a) Signed Transfer of Copyright form with manuscript

(b) Typescripts

Submitted in triplicate to any Co-editor

Double-spaced with wide margins $(e . g .30 \mathrm{~mm})$

Authors' addresses in full

Maximum of 10000 words for full articles

Maximum of 1000 words for Short Communications

(For typography see Notes for Authors, § 9)

(c) Title

Short but informative, emphasizing the structural science reported

Not to include long compound names

(d) Abstract

About 200 words

Suitable for reproduction by abstracting services without change of wording

Any references to be given in full

Sufficiently informative to obviate need for Conclusion section

Structure determination component to include: Systematic IUPAC name

Chemical formula

Formula weight

Space group

Unit-cell dimensions

Volume of unit cell $\left(\AA^{3}\right)$

$Z$

Measured and calculated densities $D_{m}, D_{x}$

Radiation and wavelength

Linear absorption coefficient

$F(000)$

Temperature of measurement

Final value of $R=\left[\sum\left(|| F_{o}|-| F_{c} \mid\right) / \sum\left|F_{o}\right|\right]$ and number of unique reflections

Principal results stated as specifically and quantitatively as possible

(e) Experimental section

Structure determination component to include:

Source of material

Crystal shape and size

Method of measuring $D_{m}$

Diffractometer used

Method of measuring intensities 
Number and $\theta$ range of reflections used for measuring lattice parameters

Absorption correction applied (with maximum and minimum values)

Maximum value of $(\sin \theta) / \lambda$ reached in intensity measurements

Range of $h, k$ and $l$

Standard reflections and their intensity variation throughout experiment

Number of reflections measured

Number of unique reflections

Value of $R_{\text {int }}\left(\sum|F-\langle F\rangle| / \sum F\right.$ from merging equivalent reflections)

Number of unobserved reflections

Criterion for recognizing unobserved reflections $[I<n \sigma(I)]$

Method used to solve structure

Definition of origin for polar structures

Independent physical measurements made to check polarity or chirality as applicable

Use of $F$ or $F^{2}$ magnitudes in least-squares refinement

Methods of locating and refining $\mathrm{H}$ atoms if applicable

Parameters refined

Values of $R, w R=\left[\sum w\left(\left|F_{o}\right|-\left|F_{c}\right|\right)^{2} / \sum w F_{o}^{2}\right]^{1 / 2}$ and $S=\left[\sum w\left(\left|F_{o}\right|-\left|F_{c}\right|\right)^{2} /(m-n)\right]^{1 / 2}$ (or the $F^{2}$ equivalents)

Method used to calculate $w$

Ratio of maximum least-squares shift to error in final refinement cycle, $(\Delta / \sigma)_{\max }$

Justification of $(\Delta / \sigma)_{\max }$ value if it exceeds 1.0

Maximum positive and maximum negative electron density in final difference Fourier synthesis, $(\Delta \rho)_{\max },(\Delta \rho)_{\min }$

Primary- and secondary-extinction value (if used)

Source of atomic scattering factors and $f^{\prime}, f^{\prime \prime}$ values

All computer programs used (see also $\S 10$ of Notes for Authors)

(f) Diagrams and photographs

Drawings in black ink or high-quality glazed prints

Cited in text

As small as possible consistent with legibility

High information density

Lettering not less than $4 \mathrm{~mm}$ high on International A4 sized paper $(210 \times 297 \mathrm{~mm})$ or $8 \frac{1}{2} \times$ 11 in (and pro rata)

Figure captions in separate list

Chemical and structural formulae preferably in camera-ready form

Stereofigures:

One per structure

Centre-to-centre separation of $55 \mathrm{~mm}$ or less

Atom labelling on left and right views that remains legible and higher than $1 \frac{1}{4} \mathrm{~mm}$ after reduction (g) Tables

Not to repeat information given in text or diagrams

Cited in text

Table number and title to be given for each

To occupy minimum space consistent with clarity

To include e.s.d.'s for all derived quantities (especially all varied parameters)

The following generally to be deposited: structure factors, anisotropic thermal parameters, leastsquares planes, unrefined $\mathrm{H}$-atom coordinates

(h) References

In form: authors' names followed by year of publication

Alphabetic order in reference list

All references in text to be given in reference list and vice-versa

Inclusive page numbers to be given in reference list

Codens-type notation with volume and initial page number to be used in multi-reference structural papers (see Table)

(i) Units and Nomenclature

SI units to be used throughout (except for $\AA$ )

Atom labels as $\mathrm{C}(1)$ etc.

Space groups in Hermann-Mauguin notation (Schönflies symbols may be used in addition for molecular symmetry)

Choice of axes as recommended by Kennard, Speakman \& Donnay [Acta Cryst. (1967). 22, 445-449]

Symmetry-equivalent atoms to be denoted as $\mathrm{C}\left(1^{\mathrm{i}}\right)$ etc., with symmetry operations defined in terms of equivalent positions

Reflections, planes, directions and forms to be unambiguously distinguished (see Notes for Authors, §8)

Chemical names and formulae to conform to IUPAC rules, including spelling of element names

Acronyms to be defined

Nomenclature of polytypes to conform to Guinier recommendations [Acta Cryst. (1984). A40, 399-404]

(j) Data to be deposited

In general:

Structure factors

Anisotropic temperature factors

Least-squares planes and deviations

Calculated $\mathrm{H}$-atom coordinates

Normal intermolecular distances

Table of non-essential bond lengths and angles

At the Co-editor's discretion:

Details of experimental procedures

Details of mathematical derivations

Lengthy mathematical appendices

Lengthy discussion not of general interest

Figures that duplicate information given elsewhere 
For macromolecular papers:

Atomic coordinates, thermal parameters and structure factors in machine-readable form with the Brookhaven Protein Data Bank [see Acta Cryst. (1981). B37, 1161-1162; Acta Cryst. (1982). B38, 1050]

For powder-data papers:

Powder data (in standard format - see Notes

for Authors, Appendix III) with the JCPDS
Format of deposited material (for all papers other than macromolecular):

Not to exceed A4 size $(210 \times 297 \mathrm{~mm})$ or $8 \frac{1}{2} \times$ 11 in

Minimum character height $1.5 \mathrm{~mm}$

Three copies, of good photocopiable quality

Codens for journals frequently referenced in crystallographic papers

\begin{tabular}{|c|c|c|c|}
\hline Acc. Chem. Res. & ACHRE4 & Chimia & CHIMAD \\
\hline ACS Symp. Ser. & ACSMC 8 & Coll. Czech. Chem. Commun. & CCCCAK \\
\hline Acta Chem. Scand. Ser. A & ACAPCT & Coord. Chem. Rev. & CCHRAM \\
\hline Acta Chem. Scand. Ser. B & ACBOCV & C.R. Séances Acad. Sci. Sér. C & CHDCAQ \\
\hline Acta Cryst. & ACCRA9 & C.R. Séances Acad. Sci. Sér. 2 & CRSUDO \\
\hline Acta Cryst. A & ACACEQ & Croat. Chem. Acta & CCACAA \\
\hline Acta Cryst. B & ASBSDK & Cryst. Lattice Defects & CLADA8 \\
\hline Acta Cryst. C & ACSCEE & Cryst. Res. Technol. & CRTEDF \\
\hline Acta Metall. & AMETAR & Cryst. Struct. Commun. & CSCMCS \\
\hline Adv. Chem. Ser. (ACS) & ADCSAJ & Curr. Sci. (India) & CUSCAM \\
\hline Adv. Inorg. Chem. Radiochem. & AICRAH & Discuss, Faraday Soc. & DFSOAW \\
\hline Adv. Struct. Res. Diffr. Methods & ASDMA9 & Dokl. Akad. Nauk SSSR & DANKAS \\
\hline Am. Mineral. & AMMIAY & Dokl. Akad. Nauk SSSR Ser. & DASKAJ \\
\hline Angew. Chem. & ANCEAD & Khim. & \\
\hline $\begin{array}{l}\text { Angew. Chem. Int. Ed. Engl. } \\
\text { Ann. Chim. (Paris) }\end{array}$ & $\begin{array}{l}\text { ACIEAY } \\
\text { ANCPAC }\end{array}$ & $\begin{array}{l}\text { Dopov. Akad. Nauk Ukr. RSR, } \\
\text { Ser. B }\end{array}$ & DANND6 \\
\hline Ann. Chim. (Rome) & ANCRAI & Eur. J. Biochem. & EJBCAI \\
\hline Ann. N.Y. Acad. Sci. & ANYAA9 & Experientia & EXPEAM \\
\hline Ann. Phys. (Leipzig) & ANPYA2 & FEBS Lett. & FEBLAL \\
\hline Annu. Rev. Phys. Chem. & ARPLAP & Ferroelectrics & FEROA8 \\
\hline Ark. Kemi & ARKEAD & Finn. Chem. Lett. & FCMLAS \\
\hline Arzneim.-Forsch. & ARZNAD & Fiz. Tverd. Tela (Leningrad) & FTVTAC \\
\hline Atti Accad. Naz. Lincei. Cl. Sci. & AANLAW & Fortschr. Mineral. & FMRLAL \\
\hline & & Gazz. Chim. Ital. & GCITA9 \\
\hline & AJCHAS & Gold Bull. & GLDBBS \\
\hline $\begin{array}{l}\text { Ber. Bunsenges. Phys. Chem. } \\
\text { Ber. Disch. Chem. Ges. }\end{array}$ & BBPCAX & Helv. Chim. Acta & $\mathrm{HCACAV}$ \\
\hline & BDCGAS & Heterocycles & HTCYAM \\
\hline $\begin{array}{l}\text { Ber. Disch. Chem. Ges. A } \\
\text { Ber. Disch. Chem. Ges. B }\end{array}$ & BDCAAA & Indian Chem. $J$. & ICLJAG \\
\hline & BDCBAD & Indian J. Chem. & IJOCAP \\
\hline $\begin{array}{l}\text { Biochem. Biophys. Res. } \\
\text { Commun. }\end{array}$ & BBRCA9 & $\begin{array}{l}\text { Indian J. Phys. } \\
\text { Indian J. Phys. Part A }\end{array}$ & $\begin{array}{l}\text { IJPYAS } \\
\text { INJADP }\end{array}$ \\
\hline Biochim. Biophys. Acta & BBACAQ & Indian J. Phys. Part B & IJPBDU \\
\hline Bioinorg. Chem. & $\mathrm{BICHBX}$ & Inorg. Chem. & INOCAJ \\
\hline Bioorg. Khim. & BIKHD7 & Inorg. Chim. Acta & ICHAA3 \\
\hline Biopolymers & BIPMAA & Inorg. Nucl. Chem. Lett. & INUCAF \\
\hline $\begin{array}{l}\text { Bull. Acad. Pol. Sci. Ser. Sci. } \\
\text { Chim. }\end{array}$ & BAPCAQ & $\begin{array}{l}\text { Int. J. Pept. Protein Res. } \\
\text { Isr. J. Chem. }\end{array}$ & IJPPC 3 \\
\hline Bull. Chem. Soc. Jpn. & BCSJA8 & $\begin{array}{l}\text { Isr. J. Chem. } \\
\text { Izv. Akad. Nauk SSSR Neorg. }\end{array}$ & $\begin{array}{l}\text { ISJCAT } \\
\text { IVNMAW }\end{array}$ \\
\hline Bull. Soc. Chim. Belg. & BSCBAG & Mater. & \\
\hline Bull. Soc. Chim. Fr. & BSCFAS & J. Am. Chem. Soc. & JACSAT \\
\hline Bull. Soc. Fr. Minéral. Cris- & BUFCAE & J. Appl. Cryst. & JACGAR \\
\hline tallogr. & & J. Biol. Chem. & $\mathrm{JBCHA3}$ \\
\hline Can. J. Chem. & CJCHAG & J. Chem. Phys. & JCPSA6 \\
\hline Can. J. Phys. & CJPHAD & J. Chem. Res. Synop. & JRPSDC \\
\hline Can. Mineral. & CAMIA6 & J. Chem. Soc. A & JCSIAP \\
\hline Carbohydr. Res. & CRBRAT & J. Chem. Soc. B & JCSPAC \\
\hline Carbon & CRBNAH & J. Chem. Soc. Chem. Commun. & JCCCAT \\
\hline $\begin{array}{l}\text { Carnegie Inst. } \\
\text { Yearb. }\end{array}$ & CIWYAO & J. Chem. Soc. Dalton Trans. & JCDTBI \\
\hline Chem. Ber. & CHBEAM & J. Chem. Soc. Perkin Trans. 1 & JCPRB4 \\
\hline Chem. Commun. & CCOMA8 & J. Chem. Soc. Perkin Trans. 2 & JCPKBH \\
\hline Chem. Commun. & CCUSBN & J. Chim. Phys. Phys. Chim. Biol. & JCPBAN \\
\hline Stockholm & & $\begin{array}{l}\text { J. Coord. Chem. } \\
\text { J. Cryst. Growth }\end{array}$ & JCCMBQ \\
\hline Chem. Erde & CERDAA & $\begin{array}{l}\text { J. Cryst. Growth } \\
\text { J. Cryst. Mol. Struct. }\end{array}$ & JCRGAE \\
\hline Chem. Ind. (London) & CHINAG & $\begin{array}{l}\text { J. Cryst. Mol. Struct. } \\
\text { J. Crystallogr. Spectrosc Res }\end{array}$ & JCMLB5 \\
\hline Chem. Lett. & CMLTAG & $\begin{array}{l}\text { J. Crystallogr. Spectrosc. Res. } \\
\text { J. Electrochem. Soc }\end{array}$ & JCREDB \\
\hline Chem. Pharm. Bull. (Jpn) & CPBTAL & J. Electrochem. Soc. & JESOAN \\
\hline Chem. Phys. Lett. & CHPLBC & J. Electron Mater. & JECMA5 \\
\hline Chem. Scr. & CSRPB 9 & J. Fluorine Chem. & JFLCAR \\
\hline Chem. Zvesti & CHZVAN & $\begin{array}{l}\text { J. Heterocycl. Chem. } \\
\text { J. Inclusion Phenom. }\end{array}$ & JHTCAD \\
\hline
\end{tabular}

J. Inorg. Biochem.

J. Inorg. Nucl. Chem.

J. Less Common Met.

J. Magn. Reson.

J. Mater. Sci.

J. Mot. Biol.

J. Mol. Spectrosc.

J. Mol. Struct.

J. Nat. Prod.

J. Nucl. Mater.

J. Org. Chem.

J. Organomet. Chem.

J. Pharmacol. Exp. Ther.

J. Phys. C

J. Phys. F

J. Phys. Chem.

J. Phys. Chem. Solids

J. Phys. Lett.

J. Phys. Soc. Jpn

J. Polym. Sci.

J. Polym. Sci. Polym. Chem. Ed.

J. Prakt. Chem.

J. Raman Spectrosc.

J. Solid State Chem

J. Struct. Chem. (Engl. Trans.)

Jpn. J. Appl. Phys.

Jpn. J. Appl. Phys. Part 1

Jpn. J. Appl. Phys. Part 2

Justus Liebigs Ann. Chem.

Khim. Prir. Soedin.

Koord. Khim.

Kristallografiya

Krist. Tech.

Life Sci.

Makromol. Chem.

Mater. Res. Bull.

Mater. Sci.

Mater. Sci. Eng.

Mineral $J$.

Mineral. Mag

Mol. Cryst. Liq. Cryst.

Monatsh Chem.

Natl. Bur. Stand. US Circ

Natl. Bur. Stand. US Monogr.

Natl. Bur. Stand. US Spec. Publ.

Natl. Bur. Stand. US Tech. Note Nature (London)

Naturwissenschaften

Neues Jahrb. Mineral. Abh.

Neues Jahrb. Mineral. Monatsh.

Nouv. J. Chim.

Organometallics

Philos. Mag.

Physica A (Amsterdam)

Physica B \& C (Amsterdam)

Physica (Utrecht)

Phys. Kondens. Mater.

Phys. Rev. B: Condens. Matter

Phys. Rev. B: Solid State

Phys. Status Solidi
JIBIDJ

JINCAO

JCOMAH

JOMRA4

JMTSAS

JMOBAK

JMOSA3

JMOSB4

JNPRDF

JNUMAM

JOCEAH

JORCAI

JPETAB

JPSOAW

JPFMAT

JPCHAX

JPCSAW

JPSLBO

JUPSAU

JPSCAU

JPLCAT

JPCEAO

JRSPAF

JSSCBI

JSTCAM

JJAPA5

JAPNDE

JAPLD8

JLACBF

KPSUAR

KOKHDC

KRISAJ

KRTEAW

LIFSAK

MACEAK

MRBUAC

MSCJDS

MSCEAA

MJTOAS

MNLMBB

MCLCA5

MOCMB7

NBSCAA

NBSMAG

XNBSAV

NB'TNAE

NATUAS

NATWAY

NJMIAK

NJMMAW

NJCHD4

ORGND7

PHMAA4

PHYADX

PHBCDQ

PHYSAG

PKOMA3

PRBMDO

PLRBAQ

PHSSAK 


\begin{tabular}{ll} 
Phys. Status Solidi A & PSSABA \\
Phys. Status Solidi B & PSSBBD \\
Pol. J. Chem. & PJCHDQ \\
Polyhedron & PLYHDE \\
Pramana & PRAMCI \\
Proc. Natl. Acad. Sci. USA & PNASA6 \\
Proc. R. Soc. London Ser. A & PRLAAZ \\
Proc. R. Soc. London Ser. B & PRLBA4 \\
Prog. Inorg. Chem. & PIOCAR \\
Prog. Med. Chem. & PMDCAY \\
Q. Rev. Chem. Soc. & QUREA7 \\
Recl: J. R. Neth. Chem. Soc. & RJRSDK \\
Recl. Trav. Chim. Pays-Bas & RTCPA3 \\
Rev. Chim. Minér. & RVCMA8 \\
Rev. Sci. Instrum. & RSINAK \\
Ric. Sci. & RISCAZ \\
Rocz. Chem & ROCHAC \\
Russ. J. Inorg. Chem. (Engl. & RJICAQ \\
\multicolumn{1}{c}{ Trans.) } &
\end{tabular}

$\begin{array}{ll}\text { S. Afr. J. Chem. } & \text { SAJCDG } \\ \text { Schweiz. Mineral. Petrogr. Mitt. } & \text { SMPTA8 } \\ \text { Science } & \text { SCIEAS } \\ \text { Solid State Commun. } & \text { SSCOA4 } \\ \text { Sov. Phys.-Crystallogr. (Engl. } & \text { SPHCA6 } \\ \text { Trans.) } & \\ \text { Sov. Phys.-Solid State (Engl. } & \text { SPSSA7 } \\ \text { Trans.) } & \\ \text { Spectrochim Acta } & \text { SPACA5 } \\ \text { Spectrochim Acta Part A } & \text { SAMCAS } \\ \text { Struct. Bonding (Berlin) } & \text { STBGAG } \\ \text { Suom. Kemisilil.B } & \text { SUKBAJ } \\ \text { Tetrahedron } & \text { TETRAB } \\ \text { Tetrahedron Lett. } & \text { TELEAY } \\ \text { Theor. Chim. Acta } & \text { TCHAAM } \\ \text { TMPM Tschermaks Mineral. } & \text { TTMMDZ } \\ \text { Petrogr. Mitt. } & \\ \text { Trans. Am. Crystallogr. Assoc. } & \text { TACAAH } \\ \text { Trans. Faraday Soc. } & \text { TFSOA4 }\end{array}$

$\begin{array}{ll}\text { Trans. Metall. Soc. AIME } & \text { TMSAAB } \\ \text { Transition Met. Chem. (N.Y.) } & \text { TRMCAM } \\ \begin{array}{l}\text { Transition Met. Chem. } \\ \text { (Weinheim) }\end{array} & \text { TMCHDN } \\ \text { Tschermaks Mineral. Petrogr. } & \text { MPMTAG } \\ \text { Mitt. } & \\ \text { Z. Anorg. Allg. Chem. } & \text { ZAACAB } \\ \text { Z. Anorg. Chem. } & \text { ZACMAH } \\ \text { Z. Elektrochem. } & \text { ZEELAI } \\ \text { Z. Kristallogr. } & \text { ZEKRDZ } \\ \text { Z. Metallkd. } & \text { ZEMTAE } \\ \text { Z. Naturforsch Teil A } & \text { ZTAKDZ } \\ \text { Z. Naturforsch. Teil B } & \text { ZNBAD2 } \\ \text { Z. Phys. Chem. (Frankfurt am } & \text { ZPCFAX } \\ \text { Main) } & \\ \text { Z. Phys. Chem. (Leipzig) } & \text { ZPCLAH } \\ \text { Zh. Neorg. Khim. } & \text { ZNOKAQ } \\ \text { Zh. Obshch. Khim. } & \text { ZOKHA4 } \\ \text { Zh. Strukt. Khim. } & \text { ZSTKAI }\end{array}$

The required form of the Codens-type notation for references in multi-reference structural papers is ACSCEE 411 (representing page 1 of Volume 41 of the journal Acta Cryst. Section C). 